\title{
An emerging role of angiotensin receptor binding protein ATRAP as a possible novel player in pathophysiology of visceral obesity and metabolic disorders
}

\author{
Kouichi Tamura, Kohji Ohki, Kengo Azushima, Kazushi Uneda, Ryu Kobayashi, Sho Kinguchi, \\ Kotaro Haruhara, Takahiro Yamaji, Takayuki Yamada, Hiromichi Wakui \\ Department of Medical Science and Cardiorenal Medicine, Yokohama City University Graduate School of Medicine, \\ Japan
}

Drug discovery targeting GPCRs is no longer limited to seeking agonists orantagonists to stimulate or block cellular responses associated with a particular receptor. GPCRs are now known to support a diversity of pharmacological profiles, a concept broadly referred to as functional selectivity. Therefore, if possible, functionally selective modulation of receptor signaling may be a safer, better tolerated, and more efficacious therapeutic strategy.

Concerning the renin-angiotensin system, while exaggerated activation of its effector AT1R promotes organ damages by BP elevation and insulin resistance via enhancement of oxidative stress and chronic inflammation, genetic inactivation of the renin-angiotensin system components, such as angiotensinogen, renin and AT1R, reportedly results in significant hypotension and provokes renal morphological and functional alteration even under baseline condition from birth, indicating that baseline AT1R signaling activity is indispensable for the maintenance of cardiovascular and renal physiology.

In the course of an investigational search for a fine means to regulate AT1R signaling at the local tissue sites, we have focused our analysis on the AT1R-associated protein (ATRAP), which is a molecule that directly binds to the carboxylterminal domain of AT1R. In contrast to the classical components of the renin-angiotensin system (i.e. angiotensinogen, renin and AT1R), alteration of ATRAP expression exerts no evident effects on baseline BP and renal morphology and function in vivo such as in ATRAP-transgenic mice and ATRAP-deficient mice in physiological condition. However, accumulating experimental results in these mice indicate that ATRAP exert s inhibitory effects on the exaggerated activation of tissue AT1R signaling in response to pathological stimuli, in order to protect cardiovascular and renal tissues under pathological conditions, in spite of no influence of ATRAP on physiological AT1R signaling.

Furthermore, we have recently suggested that the enhancement in adipose ATRAP plays a protective role against the development of diet-induced visceral obesity and insulin resistance through improvement of adipose inflammation and function via the suppression of over-activation of adipose AT1R signaling. Consequently, adipose tissue ATRAP is suggested to be an effective therapeutic target for the treatment of visceral obesity and metabolic disorders. 